\title{
Cancer risk in East Asian patients associated with acquired haemolytic anaemia: a nationwide population-based cohort study
}

\author{
Victor C. Kok ${ }^{1,2^{*}}$, Fung-Chang Sung ${ }^{3,5}$, Chia-Hung Kao ${ }^{4}$, Che-Chen Lin ${ }^{5}$ and Chun-Hung Tseng ${ }^{6}$
}

\begin{abstract}
Background: This study investigated whether patients with acquired haemolytic anaemia (AHA) would have elevated cancer risk including that for non-haematological solid tumours. We further examined whether the cancer risk would be different between patients with autoimmune type AHA (AlHA) and patients of non-AlHA.

Methods: Using nationwide population-based insurance claims data of Taiwan we identified a cohort of patients with AHA with no pre-existing cancer, $(n=3902)$ and a comparison cohort $(n=39020)$ without AHA, frequencymatched by gender, age, urbanization of residency and diagnosis date. Incidence and Cox method estimated adjusted hazard ratios (aHR) of cancers controlling covariates by the end of 2010 were calculated. Risks between patients with AlHA and non-AlHA were compared. Sensitivity analysis was carried out to measure the risk of cancer between patients with and without AHA by follow-up years.

Results: Patients with AHA had a $90 \%$ greater incidence of cancer than controls, with an aHR of 1.78 (95\% confidence interval $(\mathrm{Cl}), 1.50-2.12)]$. The overall aHRs of cancer for patients with AlHA and non-AlHA were 2.01 (95\% Cl, 1.56-2.59) and 1.87 (95\% Cl, 1.53-2.29), respectively, compared with the comparison cohort. The aHRs for lymphatic-haematopoietic malignancy were 19.5 and 9.59 in the AlHA and non-AlHA cohorts, respectively. No hazard of colorectal, lung, liver or breast cancer was significant.

Conclusions: There is a near 2-fold elevated risk for subsequent cancer in patients with AHA, particularly for lymphatic-haematopoietic malignancy, which is much greater for patients with AlHA than non-AlHA. These findings can help clinicians decide patient-centred personalized long-term management.
\end{abstract}

Keywords: Anaemia, Haemolytic, Causality, Non-autoimmune haemolytic anaemia, Retrospective cohort study, Population-based study

\section{Background}

Acquired haemolytic anaemia (AHA) is the second most prevalent haemolytic anaemia in clinical medicine after sickle cell anaemia. With respect to mechanisms, AHA can be classified on the basis of its pathogenesis: haemolysis due to intracorpuscular defects or extracorpuscular factors. Paroxysmal nocturnal haemoglobinuria $(\mathrm{PNH})$ is

\footnotetext{
* Correspondence: victorkok@asia.edu.tw

${ }^{1}$ Division of Medical Oncology, Department of Internal Medicine, Kuang Tien General Hospital, Taichung 43303, Taiwan

2Department of Biomedical Informatics, Asia University, Taichung 41354, Taiwan

Full list of author information is available at the end of the article
}

the only known AHA due to intracorpuscular defects caused by an acquired somatic mutation. Exogenous extracorpuscular factors that can cause haemolytic anaemia include autoimmune and non-autoimmune factors such as mechanical destruction, exposure to a toxic agent or drug and infections. Autoimmune haemolytic anaemia (AIHA) is the most common form of AHA in the world, excluding regions where malaria is endemic.

Recent studies have linked acquired idiopathic autoimmune haemolytic anaemia to an increased risk for future haematolymphoproliferative malignancy [1-6]. A pooled analysis of self-reported autoimmune conditions 
and the risk of non-Hodgkin lymphoma (NHL) from the InterLymph Consortium demonstrated that a personal history of haemolytic anaemia was associated with an increase in the risk for NHL [odds ratio (OR), 2.57; $95 \%$ confidence interval (CI) 1.27-5.21] [5]. In a multivariate hierarchical regression model, a population-based casecontrol study in Scandinavia showed a history of AIHA was non-significantly associated with an increased risk of Hodgkin's lymphoma with an OR of 4.5 (95\% CI, 0.8-24.7) [7].

It has been well-documented that patients with an autoimmune disease (including idiopathic AIHA) have an increased risk of malignancy although the mechanisms are still not completely clear. The underlying autoimmune disorder, with altered lymphocyte reactivity against self- or exogenous antigens are suspected to be the main cause [8-10]. Autoimmune disease may present with secondary AIHA; for example, approximately 5-10\% of patients with systemic lupus erythematosus (SLE) develop the secondary AIHA [9].

Other than idiopathic AIHA, which can be regarded as a disease entity, the remainder of AHA types, including secondary AIHA and the entire group of nonautoimmune AHA (non-AIHA), have various heterogeneous aetiologies. The occurrence of non-AIHA may be a reflection of the characteristics and severity of the parent disease. Patients with non-AIHA caused by any aetiology undergo more or less the same treatments targeting the cause of the haemolysis and its complications, and thus are exposed to potential threats from the use of corticosteroids, blood component transfusion and other therapies which may lead to altered immunity. Little is known about whether patients with non-AIHA have a similar or lesser risk for subsequent development of haematolymphoproliferative malignancies.

We postulated that there is an association between AHA and an increased risk of malignancy in the future. Moreover, there is yet a missing piece of information regarding the estimation of the risk for malignancy, particularly solid tumours, in patients with AHA. Therefore, we conducted a nationwide population-based retrospective cohort study on patients hospitalised for AHA and their subsequent cancer risk. We further differentiated the cancer risk between patients with AIHA and non-AIHA.

\section{Methods \\ Data source}

The Taiwan National Health Insurance program has been a single-payer and universal insurance program

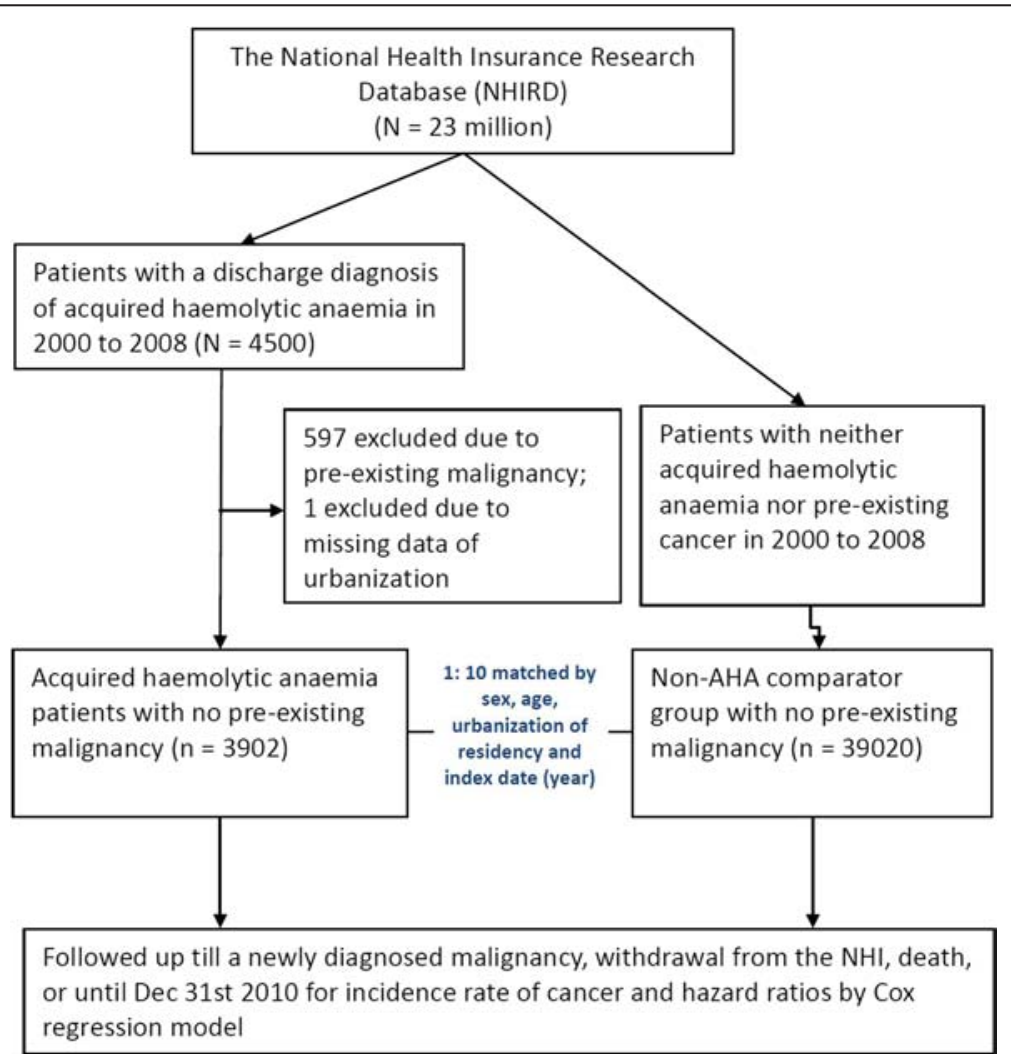

Fig. 1 Study flowchart showing steps for the selection of target populations, exclusion criteria and matching of the comparison cohort in the nationwide population-based cohort study 
Table 1 Baseline demographic data and comorbidity compared between the comparison and the acquired haemolytic anaemia (AHA) cohorts

\begin{tabular}{|c|c|c|}
\hline Variable & $\begin{array}{l}\text { Comparison cohort } \\
n=39020(\%)\end{array}$ & $\begin{array}{l}\text { AHA cohort } \\
n=3902(\%)\end{array}$ \\
\hline Age, years (SD) & $42.3(25.4)$ & $42.3(25.5)$ \\
\hline$<20$ & $8920(22.9)$ & $892(22.9)$ \\
\hline $20-39$ & $9200(23.6)$ & $920(23.6)$ \\
\hline$\geq 40$ & $20900(53.6)$ & $2090(53.6)$ \\
\hline \multicolumn{3}{|l|}{ Sex } \\
\hline Female & $22230(57.0)$ & $2223(57.0)$ \\
\hline Male & $16790(43.0)$ & $1679(43.0)$ \\
\hline \multicolumn{3}{|l|}{ Urbanization of residency } \\
\hline 1 (highest) & $9360(24.0)$ & $936(24.0)$ \\
\hline 2 & $12990(33.3)$ & $1299(33.3)$ \\
\hline 3 & $6260(16.0)$ & $626(16.0)$ \\
\hline $4+$ (lowest) & $10410(26.7)$ & $1041(26.7)$ \\
\hline \multicolumn{3}{|l|}{ Type of AHA } \\
\hline Autoimmune & 0 & $1246(31.9)$ \\
\hline Non-immune & 0 & $2656(68.1)$ \\
\hline \multicolumn{3}{|l|}{ Comorbidity } \\
\hline Diabetes & $2716(7.0)$ & 499 (12.8) \\
\hline SLE & $18(0.05)$ & $263(6.7)$ \\
\hline Alcohol-use disorder & $147(0.4)$ & $76(1.9)$ \\
\hline Splenomegaly & $25(0.1)$ & $130(3.3)$ \\
\hline CKD & $171(0.4)$ & $247(6.3)$ \\
\hline Liver cirrhosis & $161(0.4)$ & 139 (3.6) \\
\hline RA & $63(0.2)$ & $37(0.9)$ \\
\hline HBV & $108(0.3)$ & $72(1.8)$ \\
\hline $\mathrm{HCV}$ & $99(0.3)$ & $94(2.4)$ \\
\hline
\end{tabular}

Abbreviations: $A H A$ acquired haemolytic anaemia, $C K D$ chronic kidney disease, $H B V$ hepatitis $B$ virus infection, $H C V$ hepatitis $C$ virus infection, $R A$ rheumatoid arthritis, SD standard deviation, SLE systemic lupus erythematosus

since 1995 and has enrolled almost $99 \%$ of the citizens of Taiwan in 2007. The Taiwan Ministry of Health and Welfare authorized the National Health Research Institutes (NHRI) to manage all registration files and claims data and to establish the National Health Insurance Research Database (NHIRD). The NHRI created a scrambled and anonymous identification number for each insured person for linking files and to protect the privacy of patients. The NHRI gives the permission to access the data for qualified researchers. The authors have published several population-based studies on the risk of cancer in various clinical settings using the NHIRD [11-15]. This study was conducted after the approval by the Research Ethics Committee of the China Medical University, Taichung, Taiwan (CMU-REC-101012). This study was performed in accordance with the ethical standards of the 1964 Declaration of Helsinki. The informed consent was waived by the Research Ethics Committee.

For the purpose of research, specific data subsets were constructed for more timely distribution. Three relevant data subsets were chosen for this population-based study, namely, the Registry for Beneficiaries which contained each insured individual's registration data such as gender, date of birth, occupation and coverage period; the Inpatient Expenditures by Admission which included original claim data of all inpatients and finally, the Registry for Catastrophic Illness Patient Dataset (RCIPD), a unique subset of the NHIRD. Inclusion in the RCIPD required pathologic proof of malignancy, and when in doubt, the application would be examined by an independent haematologist/oncologist medical expense reviewer. Patients who satisfied the criteria for the RCIPD can benefit from a considerable reduction in out-ofpocket expenses for their cancer care throughout the country; this may also create a second check-point control from the patient side to prevent under-reporting of cancer occurrence in the RCIPD. In this research, the disease history was assembled from the Inpatient file. The disease diagnosis was recorded as per the International Classification of Diseases, Ninth Revision, Clinical Modification (ICD-9-CM).

\section{Study population}

We organized a population-based retrospective cohort study to investigate the association between AHA and subsequent cancer risk. The flow chart of the study population selection is shown in Fig. 1. The AHA cohort consisted of patients with newly-diagnosed AHA (ICD9-CM 283, from the inpatient records from 2000 to 2008. The index date was set at six months after (the first episode if more than one) the diagnosis of AHA was given at the hospital discharge. Patients with preexisting malignancy before the index date were excluded. The AHA patients were separated into two sub-cohorts: a non-AIHA sub-cohort (ICD-9-CM codes, 283.1, 283.2 and 283.9) and an AIHA sub-cohort (ICD-9-CM code = 283.0). Because of the nature of an ICD-9-CM diagnosis categorization, a drug-induced haemolytic event in a patient with glucose-6-phosphate dehydrogenase (G6PD) deficiency would be coded as inherited haemolytic anaemia and thus was not included in the studied cohort of AHA.

The comparison cohort comprised individuals who had not been diagnosed with AHA or pre-existing cancer from 2000 to 2008. In order to increase the statistical power, for each AHA patient, we randomly selected 10 comparison persons from the general population frequency-matched by age (per 5 years), gender, urbanization of residency and index date. The 


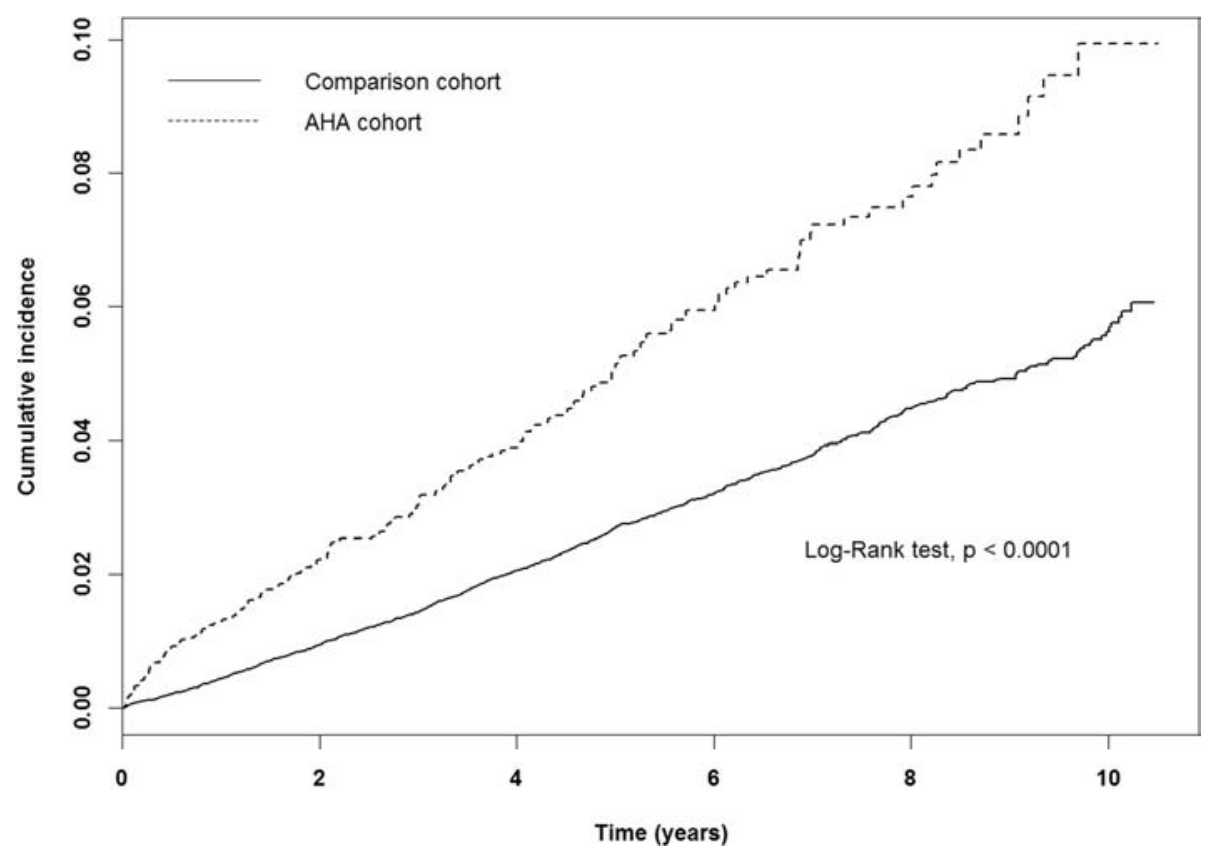

Fig. 2 The cumulative incidence of cancer in the study cohorts

index date of the comparison person was randomly matched by the same index year as that of the AHA case. The comparison cohort also had the same half year of lag observation time. We excluded all individuals with cancer diagnosed before the index date. The main parameter under consideration in this study was the incidence of developing cancer (ICD-9-CM 140208, from the RCPID). The follow-up was terminated when cancer developed, or censored when the patient withdrew from the insurance, lost to follow-up or deceased, or on 31st December 2010 (Fig. 1).

The study also collected the co-morbidity history for each study subject as confounding factors. The comorbidities before the index date included diabetes mellitus (DM, ICD-9-CM 250), alcohol use disorders (ALD, ICD-9-CM 265.2, 291, 303, 305.0, 357.5, 425.5, 535.3, 571.0, 571.1, 571.2, 571.3, 980.0 and V11.3), chronic kidney disease (CKD, ICD-9-CM 585), splenomegaly (ICD9-CM 289.4), liver cirrhosis (ICD-9-CM 571.2, 571.5 and 571.6), hepatitis B virus infection (HBV, ICD-9-CM 070.2, 070.3 and V02.61) and hepatitis $C$ virus infection (HCV, ICD-9-CM V02.62, 070.41, 070.44, 040.51 and 070.54) from the inpatient file and systemic lupus erythematosus (SLE, ICD-9-CM 710.0) and rheumatoid arthritis (RA, ICD-9-CM 714) from the RCPID. The urbanization level of residency was based on several index including population density (people $/ \mathrm{km}^{2}$ ), and population ratio of different educational levels, population ratio of elderly, population ratio of people of agriculture workers and the number of physicians per 100,000 people [16]. We categorized the urbanization of residency into 4 levels. The level 1 indicated the highest urbanization level and the level $4+$ meant the lowest level.

\section{Statistical analysis}

We compared distributions of age group, gender and comorbidities and the mean and standard deviation (SD) for age between AHA and comparison cohorts. We calculated the overall incidence density rates of cancer for both cohorts, using the total number of cancer events divided by the total sum of follow-up years for each cohort. Cox proportional hazards regression analysis was used to estimate the AHA cohort to the comparison cohort hazard ratio (HR) and $95 \%$ confidence interval. Multivariable Cox model was used to calculate the adjusted hazard ratio (aHR) and $95 \%$ confidence interval including sex, age, urbanization of residency and all comorbidities in the model. Further data analysis calculated the incidence of individual cancer and the related AHA cohort to comparison cohort aHR for major cancers, including cancers of lung (ICD-9-CM 162), liver (ICD-9-CM 155), colorectal (ICD-9-CM 153 and 154), breast (ICD-9-CM 174, only in female), lymphatic and hematopoietic tissue(ICD-9-CM 200-208) and others. We also used Kaplan-Meier method to measure and plot the cumulative incidence for both cohorts and used logrank test to examine the difference between the 2 cohorts. The proportional hazards assumption was not violated in the scaled Schoenfeld residuals test $(p=0.21)$. SAS 9.3 software (SAS Institute, Cary, NC, USA) was used to manage and analyse the data. The cumulative 
Table 2 Incidence of cancer and stratified analysis with adjusted hazard ratios by multivariate Cox proportional hazards regression analysis for study cohort

\begin{tabular}{|c|c|c|c|c|c|}
\hline Variable & Event & PYS & Rate & Crude HR (95 \% Cl) & Adjusted HR (95\% Cl) \\
\hline \multicolumn{6}{|l|}{ AHA } \\
\hline No & 1130 & 206599 & 54.7 & ref & ref \\
\hline Yes & 187 & 17912 & 104 & $1.92(1.64-2.24)$ & $1.78(1.50-2.12)$ \\
\hline Non-Autoimmune AHA & 119 & 12107 & 98.3 & $1.81(1.50-2.19)$ & $1.87(1.53-2.29)$ \\
\hline Autoimmune AHA & 68 & 5805 & 117 & $2.15(1.68-2.75)$ & $2.01(1.56-2.59)$ \\
\hline \multicolumn{6}{|l|}{ Age group } \\
\hline$<25$ & 9 & 55421 & 1.62 & ref & ref \\
\hline $25-39$ & 54 & 52892 & 10.2 & $6.32(3.12-12.8)$ & $6.78(3.35-13.7)$ \\
\hline$\geq 40$ & 1254 & 116197 & 108 & 66.9(34.7-129) & 65.37(33.9-126) \\
\hline \multicolumn{6}{|l|}{ Sex } \\
\hline Female & 606 & 127460 & 47.5 & ref & ref \\
\hline Male & 711 & 97051 & 73.3 & $1.54(1.38-1.71)$ & $1.50(1.34-1.67)$ \\
\hline \multicolumn{6}{|l|}{ Urbanization of residency } \\
\hline 1 (highest) & 282 & 53113 & 53.1 & ref & ref \\
\hline 2 & 446 & 77556 & 57.5 & $1.08(0.93-1.25)$ & $0.99(0.85-1.14)$ \\
\hline 3 & 185 & 34097 & 54.3 & $1.02(0.85-1.23)$ & $1.12(0.93-1.34)$ \\
\hline 4+ (lowest) & 404 & 59745 & 67.6 & $1.27(1.09-1.48)$ & $1.08(0.93-1.26)$ \\
\hline \multicolumn{6}{|l|}{ Comorbidity } \\
\hline \multicolumn{6}{|l|}{$\mathrm{DM}$} \\
\hline No & 1128 & 210752 & 53.5 & ref & ref \\
\hline Yes & 189 & 13759 & 137 & $2.61(2.24-3.05)$ & $1.29(1.10-1.51)$ \\
\hline \multicolumn{6}{|l|}{ SLE } \\
\hline No & 1309 & 223337 & 58.6 & ref & ref \\
\hline Yes & 8 & 1174 & 68.2 & $1.18(0.59-2.37)$ & $1.04(0.51-2.12)$ \\
\hline \multicolumn{6}{|l|}{ Alcohol $^{a}$} \\
\hline No & 1297 & 223606 & 58.0 & ref & ref \\
\hline Yes & 20 & 905 & 221 & $3.88(2.49-6.04)$ & $1.09(0.66-1.81)$ \\
\hline \multicolumn{6}{|l|}{ Splenomegaly } \\
\hline No & 1303 & 223914 & 58.2 & ref & ref \\
\hline Yes & 14 & 597 & 235 & $4.10(2.42-6.94)$ & $1.50(0.85-2.66)$ \\
\hline \multicolumn{6}{|l|}{ CKD } \\
\hline No & 1284 & 223018 & 57.6 & ref & ref \\
\hline Yes & 33 & 1493 & 221 & $3.91(2.77-5.52)$ & $1.54(1.08-2.21)$ \\
\hline \multicolumn{6}{|l|}{ Liver cirrhosis } \\
\hline No & 1278 & 223469 & 57.2 & ref & ref \\
\hline Yes & 39 & 1042 & 374 & $6.71(4.88-9.24)$ & $1.96(1.30-2.94)$ \\
\hline \multicolumn{6}{|l|}{ RA } \\
\hline No & 1314 & 224077 & 58.6 & ref & ref \\
\hline Yes & 3 & 434 & 69.1 & $1.2(0.39-3.71)$ & $0.79(0.25-2.47)$ \\
\hline \multicolumn{6}{|l|}{ HBV } \\
\hline No & 1303 & 223738 & 58.2 & ref & ref \\
\hline Yes & 14 & 773 & 181 & $3.15(1.86-5.33)$ & $1.38(0.80-2.38)$ \\
\hline
\end{tabular}


Table 2 Incidence of cancer and stratified analysis with adjusted hazard ratios by multivariate Cox proportional hazards regression analysis for study cohort (Continued)

\begin{tabular}{llllll}
\hline HCV & & & & & \\
No & 1286 & 223814 & 57.5 & ref & ref \\
Yes & 31 & 697 & 445 & $7.99(5.59-11.4)$ & $2.78(1.89-4.08)$ \\
\hline
\end{tabular}

Adjusted model was mutually adjusted

Abbreviations: $A H A$ acquired haemolytic anaemia, $A / c o h o l^{a}$ alcohol-use disorders, $C l$ confidence interval, $C K D$ chronic kidney disease, $H B V$ hepatitis $\mathrm{B}$ virus infection, $H C V$ hepatitis $C$ virus infection, $H R$ hazard ratio, $P Y$ s person-years, Rate incidence rate, per 10,000 person-years, $R A$ rheumatoid arthritis, ref reference, SLE systemic lupus erythematosus

incidence curve was plotted by SPSS. The significant level was set at less than 0.05 for two-side testing of $p$-value.

\section{Results}

We finally enrolled 3,902 AHA patients and 39,020 healthy individuals for comparison with similar mean age (42 years) and sex ratio (male: $43 \%)(p>0.05)$ in this study (Table 1 ). The proportion of AIHA in the AHA cohort was $32 \%$.

Amongst the patients with AHA, $13 \%$ had diabetes mellitus, $7 \%$ had SLE and $6 \%$ had chronic kidney disease. The proportions of the comorbidities in AHA cohort were higher than the proportions in comparison cohort $(p<0.0001)$.

The cumulative incidence of cancer after 11-year follow-up measured by Kaplan-Meier method was $3.9 \%$ greater in the AHA cohort than in the comparison cohort (log-rank test, $p<0.0001$; Fig. 2).

During a total of 17,912 patient-years for the AHA cohort under observation, 187 cancers occurred. Table 2 shows that the incidence density of cancer was 1.9-fold greater in the AHA cohort than in the comparison cohort (104 vs. 54.7 per 10,000 person-years) with an adjusted HR of 1.78 (95\% CI = 1.56-2.59) in the multivariable Cox proportional hazards regression analysis. Regarding the subtypes, compared with the individual without AHA, there were increased hazard of developing cancer for both non-AIHA patients $(\mathrm{HR}=1.87,95 \% \mathrm{CI}=$ 1.53-2.29) and AIHA patients $(\mathrm{HR}=2.01,95 \% \mathrm{CI}=$ 1.56-2.59), respectively. In this study, male gender [adjusted HR (aHR) 1.50, $95 \% \mathrm{CI}=1.34-1.67)$ ], DM (1.29,
1.10-1.51), CKD (1.54, 1.08-2.21), liver cirrhosis (1.96, 1.30-2.94) and infection with $\operatorname{HCV}(2.78,1.89-4.08)$ were significantly associated with an increased risk of cancer.

Table 3 shows the development of different types of cancer between the AHA and comparison cohorts. Overall, relative to the individuals without AHA, the patients with AHA were significantly associated with an increased risk of lymphatic and haematopoietic ( $\mathrm{HR}=$ 13.1, $95 \% \mathrm{CI}=8.46-20.3)$ and other malignant solid tumours $(\mathrm{HR}=1.82,95 \% \mathrm{CI}=1.40-2.35)$. Patients with non-AIHA and AIHA had near 10-fold (HR = 9.59, $95 \%$ $\mathrm{CI}=5.57-16.5)$ and 20 -fold $(\mathrm{HR}=19.5$, $95 \% \mathrm{CI}=11.5-$ 32.8 ) increased risk of lymphatic and haematopoietic tumours, respectively.

Table 4 shows the sensitivity analysis conducted for the risk of cancer between the AHA and comparison cohorts by follow-up years. The results suggested that patients with AHA were associated with a significantly increased risk of developing cancer as compared with individuals without AHA, although all of the study population had at least four years of follow-up.

\section{Discussion}

This nationwide population-based retrospective frequencymatched cohort study with 17,919 patient-years follow-up for the entire AHA cohort had an approximately $80 \%$ increase in the hazard for subsequent malignancy as compared with the non-AHA comparators. The randomly-selected comparison cohort was matched for age, gender, urbanization of residency and index date. Confounding factors such as type 2 diabetes, alcoholuse disorder, splenomegaly, chronic kidney disease,

Table 3 Incidence of different types of cancer and measured hazard ratios by multivariate Cox proportional hazards regression analysis for study cohorts

\begin{tabular}{lllllll}
\hline Cancer type & Liver & Lung & Colorectal & Lymphatic and haematopoietic tissue & Breast & Others \\
\hline \multirow{2}{*}{ Comparison cohort } & ref & HR $(95 \% \mathrm{Cl})$ & $\mathrm{HR}(95 \% \mathrm{Cl})$ & $\mathrm{HR}(95 \% \mathrm{Cl})$ & $\mathrm{HR}(95 \% \mathrm{Cl})$ & $\mathrm{HR}(95 \% \mathrm{Cl})$ \\
AHA cohort & $1.23(0.77-1.98)$ & $1.28(0.73-2.23)$ & $1.16(0.65-2.07)$ & $13.1(8.46-20.3)$ & ref & ref \\
Non-AlHA & $1.43(0.84-2.43)$ & $1.24(0.62-2.46)$ & $1.28(0.65-2.50)$ & $9.59(5.57-16.5)$ & $1.13(0.55-2.30)$ & $1.82(1.40-2.35)$ \\
AlHA & $0.91(0.41-2.01)$ & $1.35(0.55-3.30)$ & $0.95(0.35-2.61)$ & $19.5(11.5-32.8)$ & $1.23(0.53-2.88)$ & $1.81(1.34-2.46)$ \\
\hline
\end{tabular}

Model adjusted for age, sex, urbanization of residency, DM, SLE, alcohol-use disorders, splenomegaly, CKD, liver cirrhosis, HBV, HCV and RA

Abbreviations: $A H A$ acquired haemolytic anaemia, CKD chronic kidney disease, DM diabetes mellitus, $H B V$ hepatitis $B$ virus infection, $H C V$ hepatitis $C$ virus infection, $R A$ rheumatoid arthritis, ref reference, SLE Systemic Lupus Erythematosus 
Table 4 Sensitivity analysis showing varying estimates of the adjusted risk of developing subsequent cancer utilizing a Cox model by different cut-offs in lengthening the time lag for follow-up

\begin{tabular}{|c|c|c|c|c|c|c|c|c|}
\hline \multirow[b]{2}{*}{ Variable } & \multicolumn{3}{|c|}{ Comparison cohort } & \multicolumn{3}{|c|}{ AHA cohort } & \multirow{2}{*}{$\begin{array}{l}\text { Crude HR } \\
(95 \% \mathrm{Cl})\end{array}$} & \multirow{2}{*}{$\begin{array}{l}\text { Adjusted HR } \\
(95 \% \mathrm{Cl})\end{array}$} \\
\hline & Event & PYS & rate & Event & PYS & $\overline{\text { rate }}$ & & \\
\hline \multicolumn{9}{|c|}{ Time lag (year) } \\
\hline$>1$ & 961 & 206171 & 46.6 & 138 & 17765 & 77.7 & $1.70(1.42-2.04)$ & $1.69(1.39-2.05)$ \\
\hline$>2$ & 771 & 200747 & 38.4 & 106 & 17131 & 61.9 & $1.67(1.36-2.04)$ & $1.68(1.35-2.10)$ \\
\hline$>3$ & 610 & 187711 & 32.5 & 82 & 15680 & 52.3 & $1.66(1.32-2.09)$ & $1.70(1.32-2.19)$ \\
\hline$>4$ & 433 & 170395 & 25.4 & 61 & 13926 & 43.8 & $1.78(1.36-2.33)$ & $1.75(1.30-2.36)$ \\
\hline
\end{tabular}

Model adjusted for age, sex, urbanization of residency, DM, SLE, alcohol-use disorders, splenomegaly, CKD, liver cirrhosis, HBV, HCV and RA

Abbreviations: $A H A$ acquired haemolytic anaemia, $C l$ confidence interval, $H R$ hazard ratio, $P Y$ s person-years, rate incidence rate, per 10,000 person-years

rheumatoid arthritis, history of viral hepatitis B or $\mathrm{C}$ infection and liver cirrhosis were adjusted in the construction of the Cox model. Not only did the risk increase in patients with AIHA (aHR $=2.01)$ but it also increased in patients with non-AIHA $(\mathrm{aHR}=1.87)$ that had resulted from a group of heterogeneous aetiologies. To the best of our knowledge, this study provided for the first time the evidence for and the best estimate of the risk for subsequent cancer in patients with nonautoimmune haemolytic anaemia.

In this study, the sensitivity analysis conducted to help understand whether the risk would still persist after up to four years of follow-up demonstrated that the aHR was still around 1.75 beyond the fifth year of the followup. This sensitivity analysis helped exclude the possibility of protopathic bias (reverse causation) because some haematolymphoproliferative disorders may go unnoticed for years. In the literature, a pooled analysis of the InterLymph Consortium accrued 29,423 participants from 12 case-control studies and computed the pooled odds ratios at 2.5 (95 \% CI, 1.085.83) in a joint fixed-effect model for the future development of non-Hodgkin's lymphoma ten years after a self-reported history of haemolytic anaemia [5]. It has been noted that the use of self-reported history of haemolytic anaemia had an inherent risk for exposure misclassification bias.

The risk for lymphatic-haematopoietic malignancy was increased in both the AIHA (19.5-fold) and non-AIHA (9.6-fold) sub-cohorts in this study. Anderson et al. reported the magnitude of the association in terms of odds ratio of AIHA and chronic myeloproliferative disorder (CMPD) (excluding chronic myeloid leukaemia) to be 11.9 (4.72-30.2); however, after excluding claims within 5 years of CMPD diagnosis, the OR became statistically insignificant (OR, 4.02; $95 \% \mathrm{CI}, 0.50-32.5)$ [17]. In the same study, they also demonstrated a significant association between AIHA and acute myeloid leukaemia (OR, 3.74; $95 \%$ CI, 1.94-7.22), chronic myeloid leukaemia (OR, 5.23; 95 \% CI, 1.82-15.0), and myelodysplastic syndromes (OR, 4.12; 95 \% CI, 1.66-10.2).
This study also revealed that the risk for lymphatichaematopoietic malignancies and for certain malignant solid tumours increased. Individuals of the entire AHA cohort had an increased risk for solid tumours other than those occurring in the liver, lung, colorectal and breast.

The linkage datasets methodology utilized in the investigation of cancer incidence in this cohort study produced robust and reliable results because of the use of the unique registry dataset of severe illnesses such as cancer that offered a second check mechanism for cancer diagnosis ascertainment. It is noteworthy that this study also captured the outcomes of the risk for subsequent total cancer occurrence in individuals with diabetes mellitus (aHR 1.29; range, 1.10-1.51), chronic kidney disease (aHR 1.54; range, 1.08-2.21), liver cirrhosis (aHR 1.96; range, 1.30-2.94), and HCV infection (aHR 2.78; range, 1.89-4.08), which were in-line with the current understanding of these risks from the literature [18-22].

It may be an oversimplification to attribute the mechanisms for the positive association with future malignancies in patients with non-AIHA solely to the most feared complication resulting from prior exposure to corticosteroid therapy for controlling haemolytic anaemia and its underlying systemic disorder. The authors speculated that perhaps haemolysis itself will alter the circulating concentrations of angiogenic and pro-inflammatory markers which could contribute to the increased cancer risk [23-25].

Utilizing the coding from the discharge diagnoses to capture the occurrence of AHA and medical comorbidities has been regarded as more reliable than the use of the outpatient billing records because billings using the discharge diagnoses will go through the hands of qualified medical coding specialists [26]. Nevertheless, the potentials for inaccurate ICD-9-CM coding may exist for any administrative claims-based research. A few of the limitations of this study must be noted. Owing to the de-identified nature of each claim record in the datasets, a chart review of the patient's medical record was 
not possible. In addition, the datasets from the NHIRD did not contain biological data such as height, weight and smoking history or serial hemogram data so that the severity of haemolytic anaemia could not be determined. These limitations may potentially affect the risk estimates in this study.

\section{Conclusions}

In conclusion, the adjusted hazard ratio for lymphatichaematopoietic malignancy was elevated for 20-fold in the AIHA group and for 10-fold in the non-AIHA group. This study also provided the risk estimates for future solid tumour occurrence in patients with acquired haemolytic anaemia, particularly of malignant solid tumours other than those occurring in the lung, colorectum, liver and breast ( $80 \%$ increased risk).

\begin{abstract}
Abbreviations
AHA: acquired haemolytic anaemia; aHR: adjusted hazard ratio; AlHA: autoimmune haemolytic anaemia; CKD: chronic kidney disease; HBV: hepatitis B virus; HCV: hepatitis C virus; ICD-9-CM: international classification of diseases, ninth edition, clinical modifications; NHIRD: National Health Insurance Research Database; NHRI: National Health Research Institute Taiwan; Non-AlHA: non-autoimmune haemolytic anaemia; OR: odds ratio; PNH: paroxysmal nocturnal haemoglobinuria; RA: rheumatoid arthritis; RCPID: Registry for Catastrophic Illness Patient Dataset; SLE: systemic lupus erythematosus.
\end{abstract}

\section{Competing interests}

The authors declare that they have no competing interests.

\section{Authors' contributions}

VCK conceived the study and designed the research study. CCL performed the data sorting, merging, and applied statistical tests. VCK, FCS, and CHK contributed to the data examination, verification and manuscript revision. All authors participated in the analysis of the data, and VCK wrote the paper and gave approval for the manuscript submission.

\section{Acknowledgements}

This study is supported in part by Taiwan Ministry of Health and Welfare Clinical Trial and Research Centre of Excellence (MOHW104-TDU-B-212113002); China Medical University Hospital; Academia Sinica Taiwan Biobank Stroke Biosignature Project (BM104010092); NRPB Stroke Clinical Trial Consortium (MOST 103-2325-B-039-006); Tseng-Lien Lin Foundation, Taichung, Taiwan; Taiwan Brain Disease Foundation, Taipei, Taiwan; and Katsuzo and Kiyo Aoshima Memorial Funds, Japan. The interpretation and conclusions contained herein do not represent those of the above institutions. The authors would like to thank Enago (www.enago.com) for the English language review.

\section{Author details}

${ }^{1}$ Division of Medical Oncology, Department of Internal Medicine, Kuang Tien General Hospital, Taichung 43303, Taiwan. ²Department of Biomedical Informatics, Asia University, Taichung 41354, Taiwan. ${ }^{3}$ Department of Health Services Administration, China Medical University, Taichung 40402, Taiwan. ${ }^{4}$ Department of Nuclear Medicine and PET Centre, China Medical University Hospital, Taichung 40402, Taiwan. ${ }^{5}$ Management Office for Health Data, China Medical University Hospital, Taichung 40402, Taiwan. ${ }^{6}$ Department of Neurology, China Medical University Hospital, Taichung 40447, Taiwan.

Received: 16 October 2014 Accepted: 28 January 2016

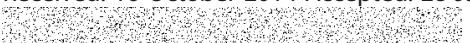

\section{References}

1. Smedby KE, Askling J, Mariette X, Baecklund E. Autoimmune and inflammatory disorders and risk of malignant lymphomas-an update. J Intern Med. 2008:264(6):514-27.

2. Sallah S, Wan JY, Hanrahan LR. Future development of lymphoproliferative disorders in patients with autoimmune hemolytic anemia. Clin Cancer Res. 2001;7(4):791-4.

3. Landgren O, Gridley G, Check D, Caporaso NE, Morris Brown L. Acquired immune-related and inflammatory conditions and subsequent chronic lymphocytic leukaemia. Br J Haematol. 2007;139(5):791-8.

4. Franks AL, Slansky JE. Multiple Associations Between a Broad Spectrum of Autoimmune Diseases, Chronic Inflammatory Diseases and Cancer. Anticancer Res. 2012;32(4):1119-36.

5. Ekstrom Smedby K, Vajdic CM, Falster M, Engels EA, Martinez-Maza O, Turner $J$, et al. Autoimmune disorders and risk of non-Hodgkin lymphoma subtypes: a pooled analysis within the InterLymph Consortium. Blood. 2008; 111(8):4029-38.

6. Anderson LA, Gadalla S, Morton LM, Landgren O, Pfeiffer R, Warren JL, et al. Population-based study of autoimmune conditions and the risk of specific lymphoid malignancies. Int J Cancer J. 2009;125(2):398-405.

7. Landgren O, Engels EA, Pfeiffer RM, Gridley G, Mellemkjaer L, Olsen JH, et al. Autoimmunity and susceptibility to Hodgkin lymphoma: a population-based case-control study in Scandinavia. J Natl Cancer Inst. 2006:98(18):1321-30.

8. Mellemkjaer L, Andersen V, Linet MS, Gridley G, Hoover R, Olsen JH. NonHodgkin's lymphoma and other cancers among a cohort of patients with systemic lupus erythematosus. Arthritis Rheum. 1997;40(4):761-8.

9. Domiciano DS, Shinjo SK. Autoimmune hemolytic anemia in systemic lupus erythematosus: association with thrombocytopenia. Clin Rheumatol. 2010; 29(12):1427-31.

10. Dey D, Kenu E, Isenberg DA. Cancer complicating systemic lupus erythematosus-a dichotomy emerging from a nested case-control study. Lupus. 2013;22(9):919-27.

11. Kao CH, Sun LM, Chen PC, Lin MC, Liang JA, Muo CH, et al. A populationbased cohort study in Taiwan-use of insulin sensitizers can decrease cancer risk in diabetic patients? Annals Oncology. 2013;24(2):523-30.

12. Kao CH, Sun LM, Liang JA, Chang SN, Sung FC, Muo CH. Relationship of zolpidem and cancer risk: a Taiwanese population-based cohort study. Mayo Clin Proc. 2012:87(5):430-6.

13. Kok VC, Horng JT, Huang JL, Yeh KW, Gau JJ, Chang CW, et al. Populationbased cohort study on the risk of malignancy in East Asian children with juvenile idiopathic arthritis. BMC Cancer. 2014;14:634.

14. Kok VC, Tsai HJ, Su CF, Lee CK. The Risks for Ovarian, Endometrial, Breast, Colorectal, and Other Cancers in Women With Newly Diagnosed Endometriosis or Adenomyosis: A Population-Based Study. Int J Gynecol Cancer. 2015;25(6):968-76.

15. Lo SF, Chang SN, Muo CH, Chen SY, Liao FY, Dee SW, et al. Modest increase in risk of specific types of cancer types in type 2 diabetes mellitus patients. Int J Cancer. 2013;132(1):182-8

16. Liu CY, Hung YT, Chuang YL, Chen YJ, Weng WS, Liu JS, et al. Incorporating development stratification of Taiwan townships into sampling design of large scale health interview survey. J Health Manag. 2006;14:1-22.

17. Anderson LA, Pfeiffer RM, Landgren O, Gadalla S, Berndt SI, Engels EA. Risks of myeloid malignancies in patients with autoimmune conditions. $\mathrm{Br}$ J Cancer. 2009;100(5):822-8.

18. Nakamura K, Wada K, Tamai Y, Tsuji M, Kawachi T, Hori A, et al. Diabetes mellitus and risk of cancer in Takayama: a population-based prospective cohort study in Japan. Cancer Sci. 2013;104(10):1362-7.

19. Sorensen HT, Friis S, Olsen JH, Thulstrup AM, Mellemkjaer L, Linet M, et al. Risk of liver and other types of cancer in patients with cirrhosis: a nationwide cohort study in Denmark. Hepatology (Baltimore, Md). 1998; 28(4):921-5.

20. Su FH, Chang SN, Chen PC, Sung FC, Huang SF, Chiou HY, et al. Positive association between hepatitis $C$ infection and oral cavity cancer: a nationwide population-based cohort study in Taiwan. PLoS One. 2012;7(10):e48109.

21. Su FH, Chang SN, Chen PC, Sung FC, Su CT, Yeh CC. Association between chronic viral hepatitis infection and breast cancer risk: a nationwide population-based case-control study. BMC Cancer. 2011:11:495.

22. Wong G, Hayen A, Chapman JR, Webster AC, Wang JJ, Mitchell P, et al. Association of CKD and cancer risk in older people. J Am Soc Nephrol. 2009; 20(6):1341-50. 
23. Maroeska Te Loo D, Bosma N, Van Hinsbergh V, Span P, De Waal R, Clarijs R, et al. Elevated levels of vascular endothelial growth factor in serum of patients with D+ HUS. Pediatric Nephrology. 2004;19(7):754-60.

24. Page AV, Tarr PI, Watkins SL, Rajwans N, Petruzziello-Pellegrini TN, Marsden PA, et al. Dysregulation of angiopoietin 1 and 2 in Escherichia coli O157:H7 infection and the hemolytic-uremic syndrome. J Infect Dis. 2013;208(6):929-33.

25. Ray P, Acheson D, Chitrakar R, Cnaan A, Gibbs K, Hirschman GH, et al. Basic fibroblast growth factor among children with diarrhea-associated hemolytic uremic syndrome. J Am Soc Nephrology. 2002;13(3):699-707.

26. Kapa S, Beckman TJ, Cha SS, Meyer JA, Robinet CA, Bucher DK, et al. A reliable billing method for internal medicine resident clinics: financial implications for an academic medical center. J Graduate Med Education. 2010;2(2):181-7.

Submit your next manuscript to BioMed Central and we will help you at every step:

- We accept pre-submission inquiries

- Our selector tool helps you to find the most relevant journal

- We provide round the clock customer support

- Convenient online submission

- Thorough peer review

- Inclusion in PubMed and all major indexing services

- Maximum visibility for your research

Submit your manuscript at www.biomedcentral.com/submit
Biomed Central 